\title{
Multivariate analysis reveals environmental and genetic determinants of element covariation in the maize grain ionome
}

Alexandra Asaro ${ }^{1}$, Brian P. Dilkes ${ }^{2}$, Ivan Baxter ${ }^{3 *}$

${ }^{1}$ Donald Danforth Plant Science Center, St. Louis, Missouri, United States of America

${ }^{2}$ Department of Biochemistry, Purdue University, West Lafayette, Indiana, United States of America

${ }^{3}$ USDA-ARS, Donald Danforth Plant Science Center, St. Louis, Missouri, United States of America

*Corresponding author

E-mail: ivan.baxter@ars.usda.gov (IB) 


\section{Abstract}

Plants obtain elements from the soil through genetic and biochemical pathways responsive

3 to physiological state and environment. Most perturbations affect multiple elements which leads

4 the ionome, the full complement of mineral nutrients in an organism, to vary as an integrated

5 network rather than a set of distinct single elements. To examine the genetic basis of covariation

6 in the accumulation of multiple elements, we analyzed maize kernel ionomes from Intermated

7 B73 x Mo17 (IBM) recombinant inbred populations grown in 10 environments. We compared

8 quantitative trait loci (QTL) determining single-element variation to QTL that predict variation

9 in principal components (PCs) of multiple-element covariance. Single-element and multivariate

10 approaches detected partially overlapping sets of loci. In addition to loci co-localizing with

11 single-element QTL, multivariate traits within environments were controlled by loci with

12 significant multi-element effects not detectable using single-element traits. Gene-by-environment

13 interactions underlying multiple-element covariance were identified through QTL analyses of

14 principal component models of ionome variation. In addition to interactive effects, growth

15 environment had a profound effect on the elemental profiles and multi-element phenotypes were

16 significantly correlated with specific environmental variables.

\section{Author Summary}

A multivariate approach to the analysis of element accumulation in the maize kernel

19 shows that elements are not regulated independently. By describing relationships between

20 element accumulation we identified new genetic loci invisible to single-element approaches. The

21 mathematical combinations of elements distinguish groups of plants based on environment,

22 demonstrating that observed variation derives from interactions between genetically controlled

23 factors and environmental variables. These results suggest that successful application of 
24 ionomics to improve human nutrition and plant productivity requires simultaneous consideration

25 of multiple-element effects and variation of such effects in response to environment.

\section{Introduction}

Elements are distinct chemical species, and studies of element accumulation frequently

28 investigate each element separately. There is overwhelming evidence, however, that element

29 accumulations covary due to physical, physiological, genetic, and environmental factors. In a

30 dramatic example in Arabidopsis thaliana, a suite of elements responds to Fe deficiency in such

31 a concerted manner that they can be used to predict the deficiency or sufficiency of Fe for the

32 plant more accurately than the measured level of Fe in plant tissues [1]. The basis of this

33 covariation can be as simple as co-transport of multiple elements. IRT1 is a metal transporter

34 capable of transporting Fe, Zn, and Mn. IRT1 is upregulated in low Fe conditions resulting in an

35 environmentally-dependent link between Fe and other ions [2]. Other pairs of co-regulated

36 elements, such as $\mathrm{Ca}$ and $\mathrm{Mg}$, which have been shown to exhibit shared genetic regulatory

37 networks in Brassica oleracea [3], should be affected identically, or predictably, by genetic

38 variation. When $A$. thaliana recombinant inbred line populations were grown in multiple

39 environments, genetic correlations among $\mathrm{Li}-\mathrm{Na}, \mathrm{Mg}-\mathrm{Ca}$, and $\mathrm{Cu}-\mathrm{Zn}$ were observed across all

40 environments while $\mathrm{Ca}-\mathrm{Fe}$ and $\mathrm{Mg}$-Fe were only correlated in a subset of environments [4].

41 Shared genetic control of ion transport without substantial environmental responsiveness should

42 result in the former pattern, along with significantly less capacity for homeostasis across

43 environmental concentrations and availabilities of elements. Environmentally-responsive

44 molecular mechanisms, reminiscent of IRT1 upregulation, could result in environmentally-

45 variable patterns of correlations. Baxter et al. previously tested element seed concentrations for

46 correlations in the maize Intermated B73 x Mo17 (IBM) recombinant inbred population, finding 
47 several correlated element pairs, the strongest of which was between Fe and $\mathrm{Zn}$ [5]. Yet, few

48 QTL impacting more than one element were found, likely due to effects on multiple elements

49 being below the threshold of observation when mapping on single element traits with limited

50 numbers of lines. These observations indicate that, while understanding the factors driving

51 individual element accumulation is important, we must consider the ionome as a network of co-

52 regulated and interacting traits [6]. We propose that formally considering this coordination

53 between elements can provide deeper insight than focusing on each element in isolation.

Multivariate analysis techniques, such as principal components analysis (PCA), can reduce

55 data dimension and summarize covariance of multiple traits as vectors of values by minimizing

56 the variances of input factors to new components. When multiple phenotypes covary, as occurs

57 for the elements in the ionome, this approach may complement single element approaches by

58 describing trait relationships. In studies on crops such as maize, PCA has been used as a strategy

59 to consolidate variables that may be redundant or reflective of a common state [7-9]. PCA has

60 proved useful in previous QTL mapping efforts, facilitating detection of new PC QTL not found

61 using univariate traits in analyses of root system architecture in rice [10] and kernel attributes,

62 ear architecture, and enzyme activities in maize [11-13]. In the current study, we expect that

63 elemental variables are functionally related and therefore need new traits to describe elemental

64 covariation. Since we do not know the exact nature of these relationships, and the ionome varies

65 depending on environment, PCA is useful in that it does not require a priori definition of

66 relationships between variables. If the PCA approach leads to novel loci and insights into how

67 the ionome is functioning, it will be a valuable addition to the study of mineral nutrient

68 regulation. 
Here we show that developing multivariate traits reveals environmental and genetic

70 effects that are not detected using single elements as traits. We performed PCA on element

71 profiles from the maize IBM population [14] grown in 10 different environments. Different

72 relationships between elements were identified that depended on environment. QTL mapping

73 using multi-element PCs as traits was carried out within each environment separately.

74 Comparing these multivariate QTL mapping results to previous QTL analyses of the same data

75 using each single element as traits for QTL analysis [15] demonstrates that a multivariate

76 approach uncovers unique loci affecting multi-element covariance. Additionally, an experiment-

77 wide PCA performed on combined data from all environments produced components capable of

78 separating lines by environment based on their whole-ionome profile. These experiment-wide

79 factors, while representative of environmental variation, also exhibited a genetic component, as

80 loci affecting these traits were detected through QTL mapping.

\section{Results}

\section{Summary of Data Collection and Previous Analysis of Single Element Traits}

We previously acquired data on 20 elements measured in the seeds from Zea mays L.

84 Intermated B73 x Mo17 recombinant inbred line (IBM) populations [14] grown in 10 different

85 location/year settings [15]. This work is briefly summarized here as it serves as the basis of our

86 comparison. The kernels came from RILs of the IBM population cultivated across six locations

87 and five years. Quantification of the accumulation of 20 elements in kernels was done using

88 inductively coupled plasma mass spectrometry (ICP-MS). Weight-adjusted element

89 measurements were used for a QTL analysis to detect loci contributing to variation in seed

90 element contents [15]. The current study is motivated by previous demonstrations of elemental

91 correlations and mutant phenotype analyses which indicate extensive relationships between 
92 elements $[1,4]$. To explore this formally, we further analyzed these data from a multiple-element

93 perspective.

94 Element to Element Correlations

Several elements were highly correlated across the dataset, exhibiting pairwise

96 relationships among lines in a given environment that passed a conservative Bonferroni

97 correction for multiple tests. We detected 209 pairs of elements that were genetically correlated

98 out of 1,900 possible correlations across environments (190 pairs per environment). We expect

99 that evidence of robust genetic control would be provided by repeated observation of trait correlations in multiple environments. Of the six locations included in this experiment, we

101 obtained data from three locations (FL, IN, and NY) from plant material grown in two different

102 years. Seven element-pairs were correlated in five or more of these six environments: Mn and

$103 \mathrm{Mg}, \mathrm{Ca}$ and $\mathrm{Sr}, \mathrm{S}$ and $\mathrm{P}, \mathrm{K}$ and $\mathrm{P}, \mathrm{P}$ and $\mathrm{Mg}, \mathrm{S}$ and $\mathrm{Mg}$, and $\mathrm{Fe}$ and $\mathrm{Zn}$ (Fig 1). Other element-

104 pair correlations were driven by the genetic variation of the IBM in fewer environments. For

105 example, Mn and P were correlated in FL05, NY05, and NY12 $\left(\mathrm{r}_{\mathrm{p}}=0.50,0.48,0.51\right)$ but were

106 not significantly correlated in FL06, IN09, or IN10 $\left(r_{p}=0.31,0.20,0.18\right)$. Thus, while some

107 correlations exist in multiple years and multiple locations, element correlations were affected by

108 both location and year.

109

In our previous single-element QTL analysis of these data, loci comprising QTL for two

110 or more different elements were detected (Table 1). This shared genetic control of multiple

111 elements was readily apparent in the trait correlations calculated within environments, as five of

112 the nine shared-element QTL exhibited corresponding element pair correlations within the given

113 environment. For example, phosphorous, which was in three of the seven most reproducible

114 element-pair correlations, exhibited the highest incidence of shared QTL with other elements. 
115 These included shared QTL between P accumulation and all three of the reproducibly P-

116 correlated elements: $\mathrm{S}$ and the cations $\mathrm{K}$ and $\mathrm{Mg}$. In addition, $\mathrm{P}$ was affected by the only QTL

117 shared between more than two elements, which affected P, S, Fe, Mn, and $\mathrm{Zn}$ accumulation in

118 NY05 (Fig 2). Consistent with the possibility of variation in transport processes affecting

119 element accumulation correlations, shared QTL were frequently found between elements with

120 similar structure, charge, and/or type, such as $\mathrm{Ca}$ and $\mathrm{Sr}$ or Fe and $\mathrm{Zn}$. These element correlations

121 and post-hoc comparisons of shared QTL localizations suggest a genetic basis for covariance of

122 the ionome in the RIL population.

123 Table 1. QTL Affecting Variation for Multiple Elements in the Same Environment.

\begin{tabular}{|l|l|l|l|l|l|l|l|}
\hline Environment & Chr & $\begin{array}{l}\text { Pos (cM) } \\
\dagger\end{array}$ & El 1 & EI 2 & EI 3 & El 4 & 12145 \\
\hline NY05 & 1 & 400 & $\mathrm{Mn}$ & $\mathrm{Ni}$ & --- & --- & --- \\
\hline NY05 & 3 & 323 & $\mathrm{Sr}$ & $\mathrm{Ca}$ & --- & --- & --- \\
\hline NY05 & 5 & 201 & $\mathrm{Mn}$ & $\mathrm{Zn}$ & $\mathrm{P}$ & $\mathrm{S}$ & $\mathrm{Fe}$ \\
\hline NY06 & 1 & 532 & $\mathrm{Mn}$ & $\mathrm{Mg}$ & --- & --- & --- \\
\hline IN09 & 4 & 306 & $\mathrm{Fe}$ & $\mathrm{K}$ & -- & --- & --- \\
\hline IN10 & 2 & 213 & $\mathrm{Mo}$ & $\mathrm{Cd}$ & --- & --- & --- \\
\hline NY12 & 5 & 203 & Zn & Fe & --- & --- & --- \\
\hline FL05 & 1 & 230 & $\mathrm{~B}$ & $\mathrm{Mn}$ & --- & --- & --- \\
\hline FL05 & 4 & 159 & $\mathrm{Fe}$ & $\mathrm{Zn}$ & --- & --- & --- \\
\hline
\end{tabular}

$125 \stackrel{+}{\dagger}$ Average position

\section{Principle Components Analysis of Covariance for Elements in the Ionome}

To better describe multi-element correlations and thereby detect loci controlling

128 accumulation of two or more elements, we derived summary values representing the covariation

129 of several elements. We implemented an undirected multivariate technique, principal

130 components analysis (PCA), for this purpose. PCA reduced correlated elements into principal

131 components (PCs), orthogonal variables that account for variation in the original dataset, each

132 having an associated set of rotations (also known as loadings) from the input variables. After

133 removing elements prone to analytical artifacts, PCA was conducted using the remaining 16

134 elements from each of the 10 environments separately. This produced 16 principal components 
135 in each environment (S1 Fig) of which we retained for further analysis only PCs representing

136 more than $2 \%$ of the total variation. This resulted in as few as 11 and as many as 15 PCs

137 depending on environment.

138 Remarkably, there is substantial overlap in the loadings of many elements in the first and

139 second PCs across some environments, suggesting a reproducible effect of genetic variation on

140 the ionome in these environments (Fig 3). Additionally, the loadings of elements are consistent

141 with the pair-wise relationships observed in the element-by-element correlations. For example,

$142 \mathrm{Ca}$ and Sr frequently load PCs in a similar direction. The PC loadings derive from inputs of

143 several elements to a single PC variable. All retained PCs in all 10 environments have a loading

144 contribution of at least .25 in magnitude from two or more elements. While some patterns existed

145 across environments, many PC loadings differed in both magnitude and direction according to

146 environment, suggesting instability of element-pair correlations across the environments. As

147 these PCs were separately calculated in each environment, we compared PC traits from different

148 environments. We used correlation tests of element loadings in PCs to identify PCs from

149 different environments that were constructed from similar relationships. Because loading

150 direction is arbitrary, both strong positive and strong negative correlations were examined. 52

151 pairs of PCs exhibited loadings correlations with a Pearson correlation coefficient greater than

1520.75 or less than -0.75 (S2 Fig). Thus, the PC analyses produced pairs of correlated PCs in

153 different locations that, while not necessarily recovered in the same order, derived from similar

154 patterns of elemental variation.

155 QTL Mapping of Ionomic Covariance Components

156 The PCs from each environment were used as traits for QTL detection. Stepwise QTL

157 mapping using these derived traits yielded 93 QTL that exceeded an estimated statistical 
158 threshold of $\alpha=0.05$ from within-environment permutations (Fig 4C). 56 of these QTL affecting

159 multiple-element covariance components overlapped with previously detected single-element

160 QTL in the same environment [15] (Fig 4A). In some cases, two or more PC traits within an

161 environment resolved to one single-element QTL. This was observed particularly for elements

162 with strong effect QTL, such as Mo, Cd, and Ni. For example, in IN10, PC2 and PC10 both have

163 QTL that co-localize with the Cd QTL on chromosome 2. Likewise, in NY05, PC3, PC5, PC6,

164 and PC9 all detect a QTL coinciding with the large-effect Ni QTL on chromosome 9. These PCs

165 within a single environment all have varying levels of Ni contribution, as well as varying levels

166 of contribution from other elements. Although the relationship among elements described by

167 each PC is distinct, the same single-element locus can be detected due to that locus affecting an

168 element that is present within each set of relationships. This repeated detection of the same

169 locations contributes to the higher number and proportion of detected PC QTL that were shared

170 with element QTL (56/93) than element QTL that were shared with PC QTL (18/79), although

171 the same genomic locations underlie this overlap. 37 PC QTL were detected at loci not seen

172 using single element traits, demonstrating that PC traits can outperform single element data for

173 the detection of shared genetic control of correlated characters. For instance, two PC5 QTL from

174 the NY06 growout were located on chromosome 1 at positions distinct from any elemental QTL

175 (Fig 4B). QTL mapping on single elements may not have the power to detect loci with small

176 coordinate effects on several elements. So as to not inflate PC-specific QTL, they are defined

177 here as QTL greater than $25 \mathrm{cM}$ away from any elemental QTL in the same environment.

179 elements within a particular environment. Of the nine loci affecting variation for multiple

180 elements in the same environment (Table 1), four loci also impact variation for a PC trait in that 
181 environment (Table 2). For example, in NY05, a QTL for PC1 overlaps the QTL that was

182 detected in the single element analyses of P, S, Fe, Mn, and Zn on chromosome 5 (Fig 2). The

183 PC QTL in this case was as strong as the association between the locus and Fe accumulation and

184 more significant than the P, S, Mn, and Zn elemental QTL. Thus, QTL mapping a multi-element

185 PC was as strong as the best single-element approach for previously detected QTL. For traits that

186 cause variation in multiple elements, such as root structure, the PC approach may be preferable

187 to single elements, particularly in cases where single element changes are of small effect or

188 below detection limits while concerted changes to multiple elements display a larger effect.

189 Table 2. QTL for Multiple Elements and PC(s) in the Same Environment.

\begin{tabular}{|l|l|l|l|l|}
\hline Environment & Chr & $\begin{array}{l}\text { Pos (cM) } \\
\dagger\end{array}$ & Elements & PC(s)190 \\
\hline NY05 & 1 & 400 & $\mathrm{Mn}, \mathrm{Ni}$ & PC11 \\
\hline NY05 & 3 & 323 & $\mathrm{Sr}, \mathrm{Ca}$ & -- \\
\hline NY05 & 5 & 201 & $\mathrm{Mn}, \mathrm{Zn}, \mathrm{P}, \mathrm{S}, \mathrm{Fe}$ & PC1 \\
\hline NY06 & 1 & 532 & $\mathrm{Mn}, \mathrm{Mg}$ & -- \\
\hline IN09 & 4 & 306 & $\mathrm{Fe}, \mathrm{K}$ & -- \\
\hline IN10 & 2 & 213 & $\mathrm{Mo}, \mathrm{Cd}$ & PC2, PC4 \\
\hline NY12 & 5 & 203 & $\mathrm{Zn}, \mathrm{Fe}$ & PC7 \\
\hline FL05 & 1 & 230 & $\mathrm{~B}, \mathrm{Mn}$ & -- \\
\hline FL05 & 4 & 159 & $\mathrm{Fe}, \mathrm{Zn}$ & -- \\
\hline
\end{tabular}

191 Average position of element QTL, PC QTL are within $5 \mathrm{cM}$

192 We compared PCs from different environments and looked for overlapping QTL among

193 PCs in different environments with correlated loadings. Of the 52 PC pairs with correlated

194 loadings, 37 had no QTL for one or both of the PCs, consistent with a shared environmental

195 factor variable in those fields as the basis of that variation. Of the remaining 15 pairs with at least

196 one QTL detected for each member of the pair, PCs in five pairs had shared QTL. In all five

197 cases, the QTL shared between these pairs of PCs correspond to a large-effect single-element

198 QTL. Six PC traits belonging to three correlated pairs, PC4 in NY05 and PC6 in IN09 ( $r_{p}=$

199 0.81), PC4 in FL05 and PC3 in NY05 $\left(r_{p}=-0.84\right)$, and PC3 in IN10 and PC2 in NC06 $\left(r_{p}=\right.$

200 0.89), detected a QTL coinciding with a Mo QTL, a locus on chromosome 1 encoding the 
201 ortholog of the A. thaliana MOT1 molybdenum transporter. The same scenario exists for PC2 in

202 IN09 and PC2 in NY05 $\left(r_{p}=-0.78\right)$, both affected by the QTL on chromosome 2 that had a

203 strong effect on Cd in our single-element QTL mapping experiments. Finally, PC8 in NC06 and

204 PC5 in NY05 $\left(r_{p}=0.76\right)$ both map to a large-effect Ni QTL. Despite the resolution to QTL

205 detected in a single-element analysis, in all of these cases correlations between loadings were not

206 driven by a single element, but rather by similar loadings for most elements (S2 Fig). In addition

207 to overlaps at these strong-effect single element QTL, 6 other pairs of correlated PCs have QTL

208 that do not overlap. Correlated PCs with QTL at different chromosomal positions in different

209 environments could be due to states, such as increased root system volume or iron deficiency,

210 that may arise from distinct processes in each environment yet can generate a consistent

211 physiological response. In these cases, the ionome displays similar trait covariance but different

212 genetic architecture consistent with genotype by environment interactions.

213 The PC approach also detected a QTL that was found for different single elements

214 depending on environment. The same locus on chromosome 7 encoded QTL for three different

215 elements, $\mathrm{Cu}, \mathrm{K}$, and $\mathrm{Rb}$, each in a different environment. $\mathrm{K}$ and $\mathrm{Rb}$ are chemical analogs.

216 Failure to detect this QTL as affecting both elements in the same environment may simply

217 indicate the poor power to detect all QTL, resulting in false negative results. It is less likely, but

218 possible, to result from incorrect assessment of a shared genetic basis due to fortuitous linkage of

219 multiple loci. Using the PC traits, we detected QTL at this position in these same three

220 environments and a fourth environment. Thus, PCs can provide an improved estimate for the

221 genetic effect on phenotypic variance for multi-element traits. In SA10, no QTL were mapped

222 for $\mathrm{Cu}, \mathrm{Rb}$, or $\mathrm{K}$ alone. Yet, this locus was detected as significantly affecting variation in PC9

223 calculated from SA10, the loadings of which show a strong contribution from $\mathrm{Cu}$ and $\mathrm{Rb}$. 
The identification of both unique and previously observed QTL through this multivariate

225

226

227

228

229

230

231

232

233

234

235

236

237

238

239

240

241

242

243

244

245

246

approach demonstrates the complementary nature of working with trait covariance as well as the component traits and supports previous work showing that elemental traits are mechanistically

interrelated. The repeated finding of results consistent with GxE led us to investigate this formally.

\section{QTL by Environment Interactions}

Our prior analyses found QTL by environment interactions contributing to accumulation of single elements [15]. Given element correlations and partially overlapping sets of element and PC QTL, we expect to detect QTL by environment interactions that impact multi-element traits.

To look at the effects of environment on genetic regulation of multi-element phenotypes, we conducted another PCA, this time on element concentrations of lines from all environments combined. If the genetic and environmental variances do not interact, we expect some PCs will reflect environmental variance and others will reflect genetic variance. However, if the ionome is reporting on a summation of physiological status that results from genetic and environmental influences, some PCs calculated from ionomic traits should be both correlated with environmental factors and result in detectable QTL.

PCA across environments. The covariance between element accumulation data across all environments was summarized using principal components analysis. Elements prone to analytical artifacts (B, Na, Al, As) were removed prior to analysis. 16 across-environment PCs (aPCs) describing the covariation of the ionome were calculated for every RIL in every environment.

Out of a concern that the different lines present in each growout unduly influenced the construction of PCs specific to each environment, we performed the following tests. First, we 
247 looked at only those locations where two or more growouts were performed, so that location

248 replication might be considered. Second, to identify a balanced sample set present in all

249 environments, we identified the lines that were grown in all of these six growouts. PCA of the 16

250 element measurements was conducted across environments (S3 Fig) and the loadings of each

251 element into each PC were recorded. Thus, the loadings of the 16 elements in the PCA were

252 calculated from a set of common genotypic checks distributed within each environment. We used

253 these loadings to calculate PCA projections (PJs) from all lines in all environments. In this way

254 we made comparisons of the same calculated values in each environment. We found that the PJS

255 and aPCs were strongly correlated; PJ1 and aPC1 were nearly identical $\left(\mathrm{r}_{\mathrm{p}}=.998\right)$ and PJs $2-5$

256 correlated with at least one of aPCs $2-5$ at $r_{p}>.66$. The correlations between the loadings from

257 PJs and aPCs reflected these same patterns. To reduce the incidence of artifacts or overfitting,

258 aPCs accounting for less than $2 \%$ of the total variation were eliminated for further analyses,

259 leaving seven aPCs.

260 Growth environment had a significant effect on all aPCs $(\mathrm{p}<0.001)$. The first two aPCs

261 were highly responsive to the environment (Fig 5). The lines from each environment cluster

262 together when plotting aPC1 vs aPC2 values, with distinct separation between environments and

263 years. In order to identify environmental factors responsible for ionome covariance, weather

264 station and soil data from all environments except SA06 were recovered from databases (see

265 methods). Correlations were calculated between season-long or quarter-length summaries of

266 temperature and the aPC values for the nine environments. The weather variables, all

267 temperature-based, were not correlated with aPCs in many cases, although correlations

268 exceeding $r_{p}=0.50$ were observed for aPCs 2,4, and 5 (Fig 6A). The strongest correlation

269 observed for aPC1 was with average maximum temperature in the fourth quarter of the growing 
270 season $\left(r_{p}=0.35\right)($ Fig $6 \mathrm{~B})$ while the highest observed for aPC2 was for average maximum

271 temperature during the third quarter $\left(r_{p}=0.58\right)($ Fig $6 C)$. The relatively small number of

272 environments, substantial non-independence of the weather variables, and likely contribution of

273 factors other than temperature limit the descriptive power of these correlations.

274 The lack of particularly strong correlations between the first two aPCs and temperature

275 variables suggests that other variables, possibly field to field variation in soil composition,

276 fertilizer application, humidity, or abiotic factors, are likely to have an influence. Correlations

277 were also calculated between environment averages of the PCs and soil variables (Fig 6D).

278 While the majority of these features were not found to be highly correlated with aPCs, we did

279 observe a strong negative correlation between aPC2 and soil $\mathrm{pH}\left(\mathrm{r}_{\mathrm{p}}=-.78\right)$ (Fig 6E).

280 In order to determine genetic effects on these components, the calculated values for aPC1

281 through aPC7 were used as traits for QTL analysis in each of the 10 environments. Unlike the

282 earlier described PCAs done in environments separately, these aPCs are calculated across all

283 environments and are therefore comparable between environments. QTL mapping detected at

284 least four loci controlling each aPC and a total of 38 QTL. Nine of these QTL were found in

285 common across multiple environments and 29 were only detected in a single environment (Fig

286 7). Of the aPC QTL, the highest LOD score QTL were present in multiple environments and

287 corresponded to the locations of the two strongest single element QTL previously detected from

288 the same data (Mo on chromosome 1 and Cd on chromosome 2). The detection of QTL, together

289 with the strong environmental determination of aPCs $1-7$, demonstrates that ionomic covariation

290 results from coordinate environmental and genetic variation.

291 Based on the stochastic detection of QTL in only a subset of growth environments,

292 substantial interaction between the environment aPC QTL is expected. A QTL of particular 
293 interest is the aPC2 QTL detected for Mo at the ortholog of the MOT1 locus. Previous studies

294 have demonstrated a connection between $\mathrm{pH}$ and molybdenum, with Mo availability in soil being

295 increased by high $\mathrm{pH}$. It was found that the MOT1 locus in A. thaliana determines response to

$296 \mathrm{pH}$ changes and resultant changes in Mo availability in an allele-specific manner, suggesting an

297 adaptive role for variation in MOT1 with respect to soil $\mathrm{pH}$ [16]. The correlation between aPC2

298 and $\mathrm{pH}$ was significant and aPC2 identified a QTL coinciding with a Mo QTL suggesting genetic

299 variation in $\mathrm{pH}-$ dependent changes to Mo availability across environments. The loading

300 magnitude for Mo into aPC2 is 0.21 but $\mathrm{Co}, \mathrm{Ni}, \mathrm{Rb}$, and $\mathrm{Cd}$ contribute even more, with loading

301 magnitudes of $0.24,0.46,0.55$, and 0.41 , respectively. QTL for aPC2 also overlap with QTL for

$302 \mathrm{Cd}$ and Ni. With aPC2 representing several elements, the correlation with soil $\mathrm{pH}$ and overlap

303 with single element QTL may reflect a multi-element phenotype responding to changes in $\mathrm{pH}$.

304 Further investigation is needed to molecularly identify the genes underlying aPC QTL, their

305 biological roles, and their interaction with specific environmental variables.

\section{Discussion}

In this study, we demonstrate that multi-trait analysis is a valuable approach for

308 understanding the ionome. The ionome is a homeostatic system, and effects on one element can

309 affect other elements [1]. Many biological processes in maize have the potential to impact

310 several elements. Indirect effects on a suite of elements have been demonstrated for numerous

311 physiological states. Radial transport of nutrients is controlled in part by endodermal suberin, the

312 structure and deposition of which can adapt in a highly plastic manner in response to deficiencies

313 in $\mathrm{K}, \mathrm{S}, \mathrm{Na}, \mathrm{Fe}, \mathrm{Zn}$, and Mn, potentially modifying transport of additional elements [17]. Other

314 examples of indirect effects can be found in Arabidopsis TSC10A mutants with reduced 3-

315 ketodihydrosphinganine (3-KDS) reductase activity. Because 3-KDS reductase is needed for 
316 synthesis of the sphingolipids that regulate ion transport through root membranes, these mutants

317 exhibit a completely root-dependent leaf ionome phenotype of increased $\mathrm{Na}, \mathrm{K}$, and $\mathrm{Rb}$, and

318 decreased $\mathrm{Mg}, \mathrm{Ca}, \mathrm{Fe}$, and $\mathrm{Mo}$ [18].

319 In line with the abundance of concerted element changes seen in ionome mutants, we

320 detected elemental correlations and QTL that were present for more than one element.

321 Phosphorous exhibited the greatest number of QTL overlap with other elements, including the

322 cations $\mathrm{K}$ and $\mathrm{Mg}$. Phosphorous is a central nutrient in plant development and regulates other

323 elements, complexing with cations in the form of phytic acid in maize seeds [19]. Additional

324 shared QTL included those between $\mathrm{Ca}$ and $\mathrm{Sr}$, Mo and $\mathrm{Mn}$, and $\mathrm{Zn}$ and Fe. $\mathrm{Ca}$ and $\mathrm{Sr}$ are

325 chemical analogs while $\mathrm{Zn}$ and Fe regulation have been linked at the physiological and

326 molecular level $[6,20]$. Mo and Mn have roles in protein assimilation and nitrate regulation [21,

327 22] and exhibit a regulatory relationship [23]. Thus, these shared QTL likely reflect genetic

328 polymorphisms affecting the activity of multi-element regulatory genes or genetic changes

329 targeted to a single element with pleiotropic effects on other elements via homeostatic

330 mechanisms.

331 The 37 PC-specific loci identify novel loci in maize with the potential to expand our

332 understanding of the genetic basis of ionome variation. Various biological mechanisms may

333 drive the detection of these unique PC QTL. For example, the ionome has been shown to exhibit

334 tissue-dependent, multi-element changes in response to nitrogen availability [24]. A unique PC

335 QTL could be detected at a nitrogen metabolism gene if variation at that gene confers additive

336 effects on multiple elements. Variation in genes involved in adaptive responses to drought stress,

337 soil nutrient deficiencies, or toxic micronutrient levels, can result in covariation among several 
338 elements without particularly strong effects on a single element $[1,6,25]$, making such genes

339 only identifiable as QTL when working with multivariate traits.

The majority of molecularly identified ionomic mutants have multi-element effects. In

341 particular, mutants in genes involved in Casparian strip function and associated root-based

342 element flow, including MYB36 [26], ESB1 [27], and LOTR1 [28], all display pleiotropic effects

343 on multiple element accumulation in the leaves. In some cases, QTL affecting these traits might

344 be detected using both single and multi-element approaches, as was the case with the

345 chromosome 5 QTL we mapped for P, S, Fe, Mn, and Zn, as well as for PC1. However, if the

346 changes to a suite of elements are small for individual elements or uncontrolled environmental

347 conditions inflate the magnitude of error in measuring the genetic effects, a multi-ionomic trait

348 may be a better fit for QTL detection. The fact that we detect both overlapping and unique sets of

349 element and PC QTL suggests that single and multivariate approaches should be used in concert

350 to avoid gaps in our understanding of element regulatory networks. The evidence suggests that

351 some of the most interesting ionome homeostasis genes, including genes that are involved in

352 environmental adaptation extending beyond the ionome, will be those best detected through

353 multivariate methods.

354 In addition to being a tool for understanding the genetics of multi-element regulation,

355 principal components also reflected environmental variation. An across-environment PCA of all

356 lines was used to find variables that describe variation between lines among all 10 environments.

357 The first two across-environment PCs capture most of the variation in the ionome across 10

358 different growouts, much of which is environmental. This can be seen in the ability of aPC1 and

359 aPC2 to separate growouts by location and, in some cases, different years within a location. 
360 Thus, components from a PCA done across environments can capture the impact of environment

361 on the ionome as a whole.

In our across-environment analysis, to account for different sets of IBM lines within

363 environments, we tested an approach of projecting loadings from a PCA on a smaller set of lines

364 onto the full data set. The similarity of the PJs and aPCs led us to conclude that the sampling

365 effects of having different subsets of lines in each environment had little effect on the trait

366 covariance estimation. This approach to validate aPCs may be useful in other studies that seek to

367 connect data from disparate experiments and federate data collected by multiple laboratories.

368 The method of deriving traits across environments using a small set of genotypic checks opens

369 up the possibility of using multi-trait correlations across environments to permit very large scale

370 GxE mapping experiments on data sets not initially intended for this purpose. Retrospective

371 analysis of data, or further data generation from preexisting biological material present in both

372 public and private spheres, is enabled by this approach. For example, multiple association panels

373 have been constructed for trait mapping in maize. Typically, comparison of multi-trait

374 correlations across different populations is inhibited by our inability to ensure the 1:1

375 correspondence of traits. By using the subset of lines common to all mapping populations to

376 create a projection, comparable traits could be reflected onto to full datasets for comprehensive

377 genetic evaluation and the loci detected in each panel could then be compared, as we have done 378 here.

PCA on all environments is a way to find variation resulting from environmental factors

380 that impact multiple elements, for example weather or soil variables. The weather data available

381 to us for this study was limited to maximum and minimum temperature. We observed the

382 strongest correlations for aPC1 and aPC2 during the third and fourth quarters of the growing 
season. Because seed filling occurs in the latter part of the season, temperature during this time could have a pronounced effect on seed elemental composition. However, the lack of striking correlations between environmental components and the projections and aPCs, environmental

386 factors other than temperature must be the strongest factors. Information on soil properties

387 provided insight into a potential driver of the environmental variability captured by aPC2, with a

388 strong negative correlation between aPC2 and soil pH. Soil pH alters element availability in soil, 389 and $\mathrm{pH}$ differences between locations should result in different kernel ionomes. QTL were mapped to the aPCs that describe whole ionome variation across

391 environments. These loci may encompass genes that pleiotropically affect the ionome in an

392 environmentally-responsive manner. The correlation between aPC2 with $\mathrm{pH}$ as well as the

393 finding of an aPC2 QTL for Mo exemplifies the possibility of using across-environment PCA to

394 detect element homeostasis loci that respond to a particular environmental or soil variable and

395 produce a multi-element phenotype. To the extent that these differences are adaptive, these

396 alleles can contribute to local adaptation to soil environment and nutrient availability. The

397 identification of aPC QTL indicates that the variation captured by aPCs has both environmental

398 and genetic components. Our previous study using single element traits found extensive GxE in

399 this dataset through formal tests, so it is not surprising that we see a large environmental

400 component as well as genetic factors contributing to variation in the across-environment PCs.

401 Experiments with more extensive weather and soil data, or carefully manipulated environmental

402 contrasts, are needed to create models with additional covariates and precisely represent

403 environmental impacts. This multivariate approach could be especially powerful in studies with

404 extensive and consistent environmental variable recording, such as the "Genomes to Fields" 
405 Initiative, where specific environmental variables could be included in QTL models of multi-

406 element GxE.

\section{Conclusions}

Here we have shown that treating the ionome as an interrelated set of traits using PCA

409 within environments can identify novel loci. PCA across environments allowed us to derive traits

410 that described both environmental and genetic variation in the ionome.

\section{Methods}

\section{Field Growth and Data Collection}

413 Field growth and elemental profile analysis. Lines belonging to the Intermated B73 x Mo17

414 recombinant inbred (IBM) population [14] were grown in 10 different environments: Homestead,

415 Florida in 2005 (220 lines) and 2006 (118 lines), West Lafayette, Indiana in 2009 (193 lines) and

4162010 (168 lines), Clayton, North Carolina in 2006 (197 lines), Poplar Ridge, New York in 2005

417 (256 lines), 2006 (82 lines), and 2012 (168 lines), Columbia, Missouri in 2006 (97 lines), and

418 Ukilima, South Africa in 2010 (87 lines). Elemental analysis was carried out in a standardized

419 inductively coupled plasma mass spectrometry (ICP-MS) pipeline previously described in detail

420 [15]. Analytical outlier removal and weight normalization was performed following data

421 collection as described in our previous analysis of these data.

\section{Computational Analysis}

423 Element correlation analysis. Within environments, 190 Pearson correlation coefficients were

424 calculated, one for each pair of the 20 measured elements. To control for multiple tests, we

425 applied a Bonferroni correction at an alpha level of 0.05. Given 190 possible combinations,

426 correlations with a p-value below $0.05 / 190=0.00026$ were regarded as significant.

427 Principal components analysis of ionome variation within environments. Elements prone to 
428 analytical error (B, Na, Al, As) were removed before to PC analysis, leaving 16 elements: Mg, P,

$429 \mathrm{~S}, \mathrm{~K}, \mathrm{Ca}, \mathrm{Mn}, \mathrm{Fe}, \mathrm{Co}, \mathrm{Ni}, \mathrm{Cu}, \mathrm{Zn}, \mathrm{Se}, \mathrm{Rb}, \mathrm{Sr}, \mathrm{Mo}$, and $\mathrm{Cd}$. In an attempt to summarize the effects

430 of genotype on covariance of ionomic components, a PCA was done using elemental data for

431 each of the 10 environments separately. The prcomp function in $\mathrm{R}$ with scale $=$ TRUE was used

432 for PCA on elemental data to perform PCA on the line average element values in an

433 environment. This function performs singular value decomposition on a scaled and centered

434 version of the input data matrix, computing variances with the divisor $N-1$. 16 PCs were returned

435 from each environment. After removal of PCs accounting for less than $2 \%$ of the variance, the 10

436 sets of PCs were used as traits in QTL analysis. Variance proportions and trait loadings for all

437 PCs calculated across 10 environments are provided in S1 Table.

438 QTL Mapping: principal components. QTL mapping was done using stepwise forward-

439 backward regression in R/qtl [29] as described previously for element phenotypes [15]. The

440 mapping procedure was done for each environment separately, with PC line means for RILs in

441 the given environment as phenotypes and RIL genotypes as input. The stepwiseqtl function was

442 used to produce an additive QTL model for each PC, with the max number of QTL allowed for

443 each trait set at 10 . The $95^{\text {th }}$ percentile LOD score from 1000 scanone permutations was used as

444 the penalty for addition of QTL. The QTL model was optimized using refineqt for maximum

445 likelihood estimation of QTL positions. The locations of the PC QTL detected in this study were

446 compared to the single element QTL from our previous study. Loci were considered distinct if

447 they were at least $25 \mathrm{cM}$ away from any single element QTL detected in the environment in

448 which the PC QTL was detected. This serves as a conservative control in order to minimize the

449 mistaken assessment of novelty for QTL with small changes in peak position.

450 QTL by environment analysis: PCA across environments. The 16 most precisely measured 
451 elements were used for an additional principal components analysis. Again, the prcomp function

452 in R with scale $=$ TRUE was used for PCA on elemental data, however, all 16 element

453 measurement values in all lines in all of the 10 environments were combined into one PCA.

454 These PCs are referred to as across-environment PCs (aPCs). The first 7 aPCs explained 93\% of

455 the total covariation of these traits. A linear model was used to test the relationship of

456 environmental parameters on these aPCs. All seven aPCs were also used for stepwise QTL

457 mapping by the same method described above.

458 QTL by environment analysis: Projection-PCA across environments. The sets of lines grown

459 in each our ten environments were drawn from the same population [14] but different subsets

460 were grown and harvested in different environments. To achieve common multivariate

461 summaries for all lines and growouts, we performed an alternative PCA using a smaller set of

462 common lines. We then projected the loadings from this PCA onto the full dataset, as follows.

463 First, a PCA was conducted on 16 lines common to six of the 10 environments (FL05, FL06,

464 IN09, IN10, NY05, NY12). The loadings for each PC from this PCA were then used to calculate

465 values from full set of lines across 10 environments to generate PCA projections (PJs). These

466 derived values based on a common-line PCA were compared to previously described aPC values

467 from the PCA done on all lines at once. Correlations between PJs and aPCs were computed to

468 compare the outcomes of the two methods.

469 Weather and soil data collection and analysis. Weather data for FL05, FL06, IN09, IN10,

470 NC06, NY05, NY06, and NY12 was downloaded from Climate Data Online (CDO), an archive

471 provided by the National Climatic Data Center (NCDC) through the National Oceanic and

472 Atmospheric Administration (http://www.ncdc.noaa.gov/cdo-web/). Data were not available for

473 the South Africa growout. Daily summary data for each day of the growing season were 
474 tabulated from the weather station nearest to the field location. Weather stations used to obtain

475 data for each location are indicated in S2 Table. Minimum temperature (in degrees Celsius) and

476 maximum temperature (in degrees Celsius) were available in each location. With these variables,

477 average minimum temperature, and maximum temperature were calculated across the 120-day

478 growing season as well as for 30 day quarters. Growing degree days (GDD) were calculated for

479 the entire season and quarterly using the formula GDD $=((\operatorname{Tmax}+\operatorname{Tmin}) / 2)-10$.

480 Data describing soils from each location were obtained from the Web Soil Survey provided

481 by the USDA Natural Resources Conservation Service

482 (http://websoilsurvey.sc.egov.usda.gov/App/HomePage.htm). A representative area of interest

483 was selected at the site of plant growth using longitude and latitude coordinates. When an area

484 contained more than one soil type, a weighted average of measurements from all soil types was

485 used. The data we downloaded from the Web Soil Survey were: $\mathrm{pH}$, electrical conductivity (EC)

486 (decisiemens per meter at 25 degrees C), available water capacity (AWC) (centimeters of water

487 per centimeter of soil), available water supply (AWS) (centimeters), and calcium carbonate

488 ( $\mathrm{CaCO} 3)$ content (percent of carbonates, by weight). Layer options were set to compute a

489 weighted average of all soil layers.

The relationships between the seven experiment wide aPCs and the weather and soil

491 variables were estimated by calculating Pearson correlation coefficients for the pairwise

492 relationships. Correlations were also calculated between average element values and soil and

493 weather variables in each environment.

\section{Acknowledgements}

495 The authors would especially like to thank our field collaborators Sherry Flint-Garcia, Peter

496 Balint-Kurti, Torbert Rocheford, Jonathan Lynch, and Robert Snyder for their dedicated efforts

497 to provide the seeds analyzed for this project. This work was supported by funding from the 
National Science Foundation (IOS-1126950, IOS-1450341), the USDA Agricultural Research Service (5070-21000-039-00D). AA was a recipient of a Danforth Plant Science Fellowship from the Donald Danforth Plant Science Center.

\section{References}

1. Baxter IR, Vitek O, Lahner B, Muthukumar B, Borghi M, Morrissey J, et al. The leaf ionome as a multivariable system to detect a plant's physiological status. Proceedings of the National Academy of Sciences. 2008;105: 12081-12086.

2. Korshunova YO, Eide D, Clark WG, Guerinot ML, Pakrasi HB. The IRT1 protein from Arabidopsis thaliana is a metal transporter with a broad substrate range. Plant molecular biology. 1999; 40: 37-44.

3. Broadley MR, Hammond JP, King GJ, Astley D, Bowen HC, Meacham MC, et al. Shoot calcium and magnesium concentrations differ between subtaxa, are highly heritable, and associate with potentially pleiotropic loci in Brassica oleracea. Plant Physiol. 2008;146: 1707-1720.

4. Buescher E, Achberger T, Amusan I, Giannini A, Ochsenfeld C, Rus A, et al. Natural genetic variation in selected populations of Arabidopsis thaliana is associated with ionomic differences. PLoS One. 2010;5: e11081.

5. Baxter IR, Gustin JL, Settles AM, Hoekenga OA. Ionomic characterization of maize kernels in the intermated B73 x Mo17 population. Crop Science. 2013;53: 208-220.

6. Baxter I. Ionomics: studying the social network of mineral nutrients. Current Opinion in Plant Biology. 2009;12: 381-386.

7. Burton AL, Johnson J, Foerster J, Hanlon MT, Kaeppler SM, Lynch JP, et al. QTL mapping and phenotypic variation of root anatomical traits in maize (Zea mays L.). Theoretical and Applied Genetics. 2015;128: 93-106.

8. Bouchet S, Bertin P, Presterl T, Jamin P, Coubriche D, Gouesnard B, et al. Association mapping for phenology and plant architecture in maize shows higher power for developmental traits compared with growth influenced traits. Heredity. 2017;118: 249-259.

9. Frey FP, Presterl T, Lecoq P, Orlik A, Stich B. First steps to understand heat tolerance of temperate maize at adult stage: identification of QTL across multiple environments with connected segregating populations. Theoretical and Applied Genetics. 2016;129: 945-961.

10. Topp CN, Iyer-Pascuzzi AS, Anderson JT, Lee C-R, Zurek PR, Symonova O, et al. 3D phenotyping and quantitative trait locus mapping identify core regions of the rice genome controlling root architecture. Proceedings of the National Academy of Sciences. 2013;110: E1695-E1704.

11. Liu Z, Garcia A, McMullen MD, Flint-Garcia SA. Genetic Analysis of Kernel Traits in Maize-Teosinte Introgression Populations. G3: Genes Genomes Genetics. 2016;6: 2523.

12. Choe E, Rocheford TR. Genetic and QTL analysis of pericarp thickness and ear architecture traits of Korean waxy corn germplasm. Euphytica. 2012;183: 243-260.

13. Zhang N, Gibon Y, Gur A, Chen C, Lepak N, Hohne M, et al. Fine Quantitative Trait Loci Mapping of Carbon and Nitrogen Metabolism Enzyme Activities and Seedling Biomass in the Intermated Maize IBM Mapping Population. Plant Physiology. 2010; 154: 1753-1765.

14. Lee M, Sharopova N, Beavis WD, Grant D, Katt M, Blair D, et al. Expanding the genetic map of maize with the intermated B73 x Mo17 (IBM) population. Plant molecular biology. 
2002;48: 453-461.

15. Asaro A, Ziegler G, Ziyomo C, Hoekenga OA, Dilkes BP, Baxter I. The Interaction of Genotype and Environment Determines Variation in the Maize Kernel Ionome. G3: Genes Genomes Genetics. 2016;6: 4175-4183.

16. Poormohammad Kiani S, Trontin C, Andreatta M, Simon M, Robert T, Salt DE, et al. Allelic Heterogeneity and Trade-Off Shape Natural Variation for Response to Soil Micronutrient. PLOS Genetics. 2012;8: e1002814.

17. Barberon M, Vermeer J, De Bellis D, Wang P, Naseer S, Andersen T, et al. Adaptation of Root Function by Nutrient-Induced Plasticity of Endodermal Differentiation. Cell. 2016;164: 447-459.

18. Chao DY, Gable K, Chen M, Baxter I, Dietrich CR, Cahoon EB, et al. Sphingolipids in the Root Play an Important Role in Regulating the Leaf Ionome in Arabidopsis thaliana. Plant Cell. 2011;23: 1061-1081.

19. Lopez-Arredondo DL, Leyva-Gonzolez MA, Gonzolez-Morales SI, Lopez-Bucio J, Herrera-Estrella L. Phosphate nutrition: improving low-phosphate tolerance in crops. Annual Review of Plant Biology. 2014;65: 95-123.

20. Lin Y-F, Liang H-M, Yang S-Y, Boch A, Clemens S, Chen C-C, et al. Arabidopsis IRT3 is a zinc-regulated and plasma membrane localized zinc/iron transporter. New Phytologist. 2009; 182: 392-404.

21. Mulder EG. Importance of molybdenum in the nitrogen metabolism of microorganisms and higher plants. Plant and Soil. 1948;1: 94-119.

22. Mulder EG, Gerretsen FC. Soil manganese in relation to plant growth. Adv Agron. 1952;4: 221-277.

23. Millikan CR. Antagonism between molybdenum and certain heavy metals in plant nutrition. Nature. 1948;161: 528.

24. Chu Q, Watanabe T, Shinano T, Nakamura T, Oka N, Osaki M, et al. The dynamic state of the ionome in roots, nodules, and shoots of soybean under different nitrogen status and at different growth stages. 2016;17: 488-498.

25. Baxter I, Dilkes BP. Elemental Profiles Reflect Plant Adaptations to the Environment. Science. 2012;336: 1661-1663.

26. Kamiya T, Borghi M, Wang P, Danku JMC, Kalmbach L, Hosmani PS, et al. The MYB36 transcription factor orchestrates Casparian strip formation. Proceedings of the National Academy of Sciences. 2015;112: 10533-10538.

27. Baxter I, Hosmani PS, Rus A, Lahner B, Borevitz JO, Muthukumar B, et al. Root suberin forms an extracellular barrier that affects water relations and mineral nutrition in Arabidopsis. PLoS Genet. 2009;5: e1000492.

28. Li B, Kamiya T, Kalmbach L, Yamagami M, Yamaguchi K, Shigenobu S, et al. Role of LOTR1 in Nutrient Transport through Organization of Spatial Distribution of Root Endodermal Barriers. Current Biology. 2017;27: 758-765.

29. Broman KW, Speed TP. A model selection approach for the identification of quantitative trait loci in experimental crosses. Journal of the Royal Statistical Society: Series B (Statistical Methodology). 2002;64: 641-656. 


\section{Supporting Information}

S1 Fig. Variances of Principal Components from PCA within 10 Environments. Eigenvalues (amount of variation explained) for each PC are shown on the y-axis. Lines are colored by environment.

S2 Fig. Loadings of Principal Components from Different Environments. Loadings for each element are plotted for PCs from different environments. Loadings of PCs plotted on the same graph are correlated as indicated. PCs shown in (A), (B), and (C) all have a QTL coinciding with Mo QTL on chromosome 1. PCs shown in (D) have a QTL coinciding with Cd QTL on chromosome 2. PCs shown in (E) have a QTL coinciding with Ni QTL on chromosome 9.

\section{S3 Fig. Variances of Principal Components from PCA on Lines from all Environments. Eigenvalues (amount of variation explained) for each aPC are shown on the y-axis.}

S4 Fig. aPC1 and aPC2 Loadings Biplot. PCA plots showing aPC1 and aPC2 loadings. Variance explained for each $\mathrm{PC}$ is indicated along axes.

\section{S1 Table. PC Variance Proportions and Loadings Across 10 Environments.}

\section{S2 Table. Weather Station Locations.}

\section{Figure Legends}

Fig 1. Element Correlations Diagrams for Locations with Repeated Measurements. Pairwise correlations of 20 kernel elements in varying environments, shown for the experiments within locations having data from multiple years (FL, IN, and NY). Correlations were calculated as the Pearson correlation coefficient $\left(r_{p}\right)$ between concentration values for each element pair. Significance was evaluated using a Bonferroni correction for multiple tests within each environment and set at a corrected $\mathrm{p}$ value of 0.05 . Lines between elements represent significant pairwise correlations, weighted by strength of correlation. Positive and negative correlations are represented as solid and dashed lines, respectively. Red lines indicate correlations present in at least 5 of the 6 environments shown.

Fig 2. Multiple Element QTL. Stepwise QTL mapping output from the NY05 population for P, $\mathrm{S}, \mathrm{Fe}, \mathrm{Mn}, \mathrm{Zn}$, and $\mathrm{PC} 1$. Position in $\mathrm{cM}$ on chromosome 5 is plotted on the $\mathrm{x}$-axis and LOD score is shown on the $y$-axis. $95^{\text {th }}$ percentile of highest LOD score from 1000 random permutations is indicated as horizontal line.

Fig 3. PCA Plots in Multiple Environments. PCA plots showing PC1 and PC2 loadings in different years in three locations (FL, IN, and NY). PC1 and PC2 values for each line are plotted as points and PC1 and PC2 loadings of each element are indicated by blue arrows. The data for different years for each of three locations, FL, IN, and NY are plotted. The percent of total variation explained by each $\mathrm{PC}$ is labeled on the axes. 
Fig 4. Principal Component QTL from 10 environments. PCs were derived from elemental data separately in each of 10 environments and used as traits for QTL mapping. (A) 172 total element and PC QTL were mapped. The two boxes represent the 79 and 93 elemental and PC QTL, respectively. 18 element QTL overlap with PC QTL from the same environment. 56 PC QTL overlap with element QTL from the same environment. Sets of non-unique QTL are shown in the center box. QTL unique to elements, 61, and to PCs, 37, are shown outside of the shared box. (B) QTL mapping output for PC5 from the NY06 population. Position on chromosome 1 is shown on the x-axis, LOD score is on the y-axis. All significant NY06 element QTL on chromosome 1 are shown in grey $(\alpha=0.05)$. Two PC5 QTL, at 169.7 and $271.2 \mathrm{cM}$, are unique to PC5 and do not overlap with any elemental QTL. A PC5 QTL at $379.7 \mathrm{cM}$ is shared with a molybdenum QTL. (C) Significant PC QTL $(\alpha=0.05)$ for PCs in 10 environments. QTL location is shown across the 10 chromosomes on the $\mathrm{x}$-axis. Environment in which QTL was found is designated by color. QTL are represented as dashes of uniform size for visibility. Four regions highlighted in grey represent the four loci found for multiple PC traits in multiple environments $(>2)$.

Fig 5. PCA Separates Lines by Environment. PC1 and PC2 separate lines by environment. Points correspond to lines, colored by their environment. (A) Across-environment PC1 vs PC2 values for each line, colored by environment. Percentage of total variance accounted for by each PC indicated on the axes. (B) Average across-environment PC1 vs PC2 values for all lines in each environment.

Fig 6. aPC and Weather Variable Correlations. (A) Heatmap showing Pearson correlation coefficients $\left(r_{p}\right.$ between averaged aPC 1-7 values across environments and averages for maximum temperature, minimum temperature, and GDD across the growth season and for each quarter of the season. Red and blue intensities indicate strength of positive and negative correlations, respectively. (B) Average aPC1 values for 9 environments vs. average maximum temperature for each environment over the fourth quarter of the growing season. Points colored by environment. Pearson correlation coefficient is shown within the graph. (C) Average aPC2 values for nine environments vs. average maximum temperature for each environment over the 3rd quarter of the growing season. (D) Heatmap showing correlations between aPCs 1-7 and soil attributes: $\mathrm{pH}$, electrical conductivity (EC), available water capacity (AWC), available water storage (AWS), and calcium carbonate (CaCO3). (E) Average aPC2 values vs. $\mathrm{pH}$.

Fig 7. Across-Environment PCA QTL in 10 Environments. QTL identified for across environment PCA traits (aPCs 1-7). (A) Total number of QTL detected for each aPC, colored by environment. (B) Significant QTL $(\alpha=0.05$ ) for aPCs $1-7$. QTL location is shown across 10 chromosomes (in cM) on the X-axis. Dashes indicate QTL, with environment in which QTL was found designated by color. All dashes are the same length for visibility. 


\section{Elements and PC1 Share QTL from NY05}

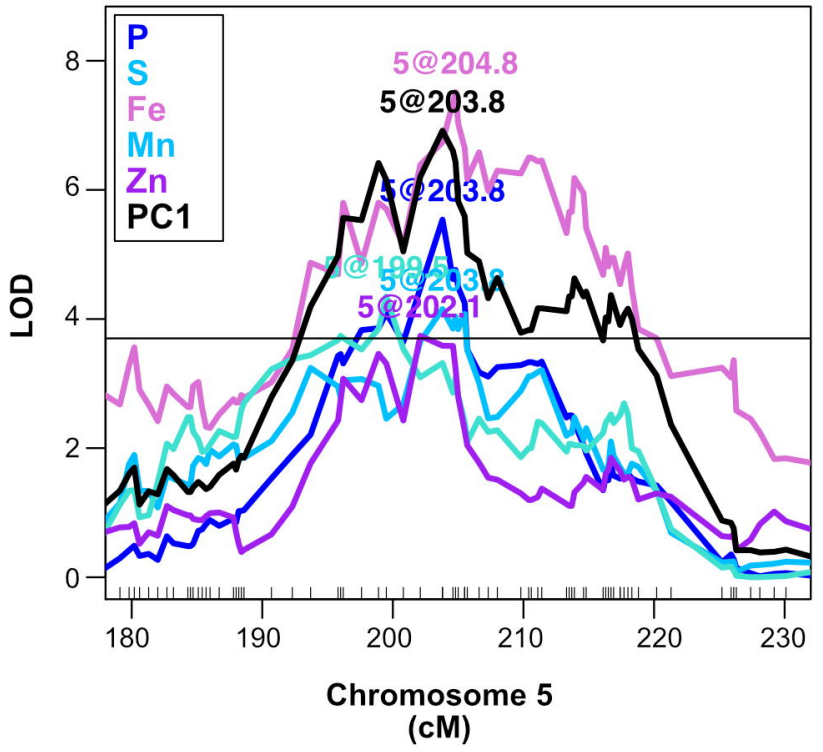




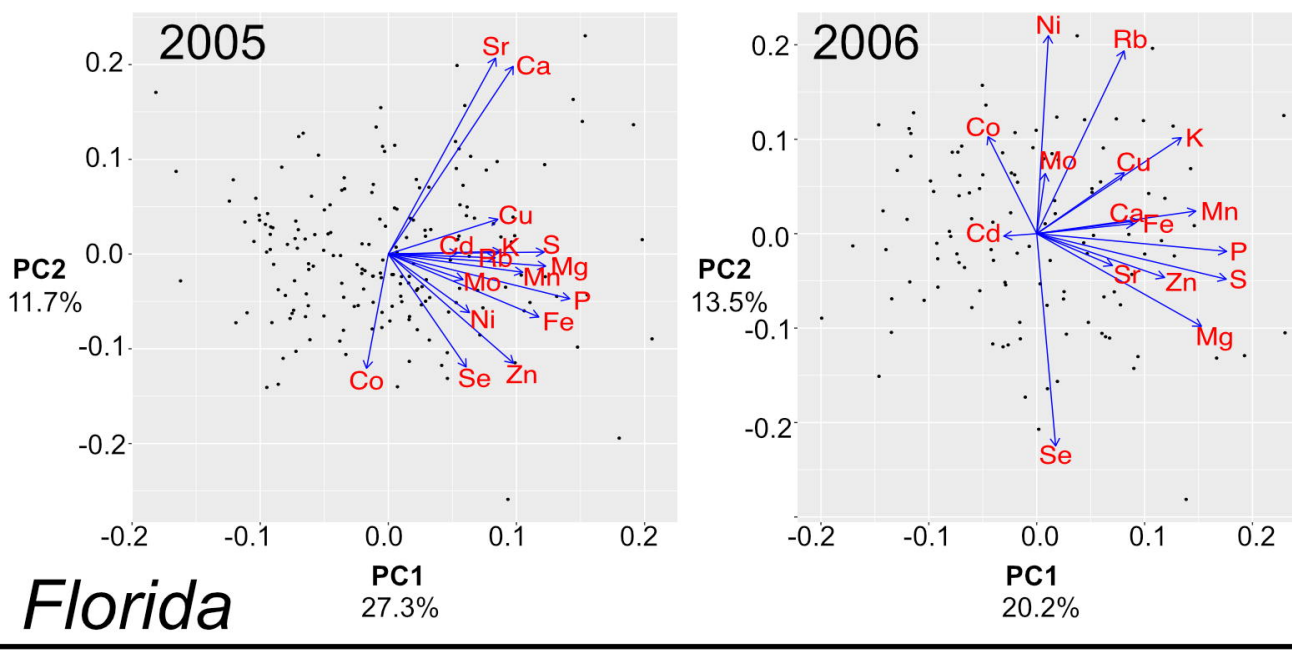

Florida $\quad \underset{27.3 \%}{\mathrm{PC} 1}$

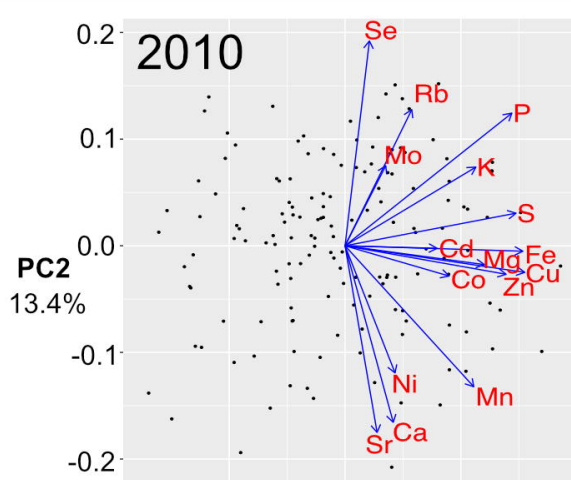

$-0.2$
0.2
0.1
$\begin{array}{lll}0.0 & -0.1 & -0.2\end{array}$
Indiana
0.1

$0.0^{-0.1}-0.2$
PC1
$20.5 \%$

2005

0.3. 2012

0.1 .

PC2

$12.9 \%$

$-0.1$

$-0.2$

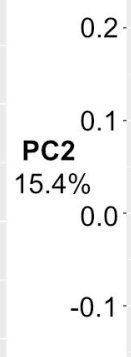

0.2
R $\mathrm{R} b$

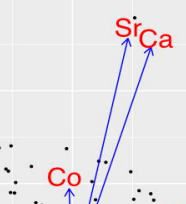
$-0.2$
$-0.2$
$-0.1$
0.0
0.1
0.2
PC1
0.0
0.1
0.2

New York ${ }^{24.8 \%}$ 
A

180 total QTL detected using complementary methods

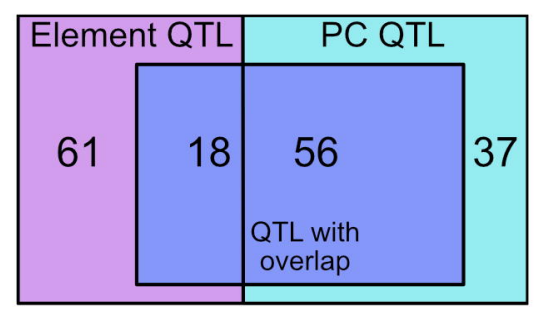

C

B Unique and Overlapping PC5 QTL in NY06

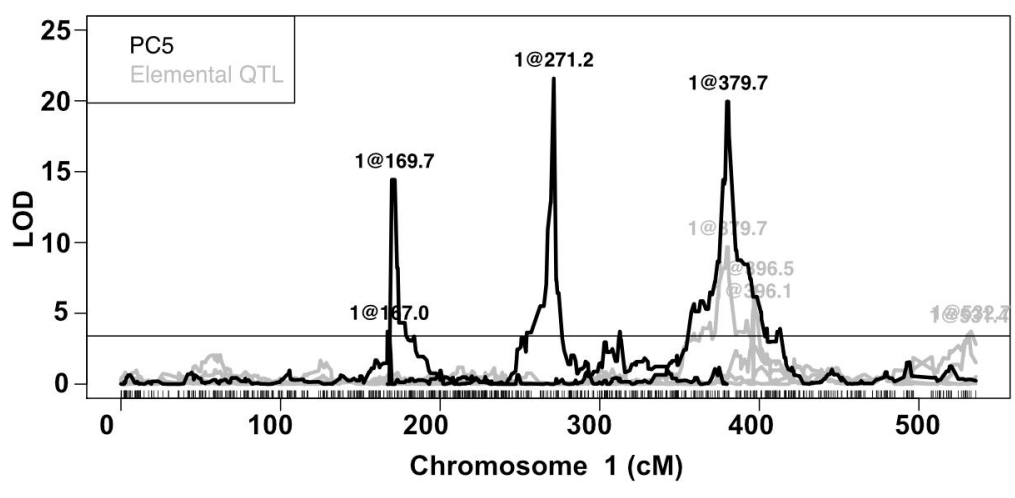

Full Genome View: PC QTL

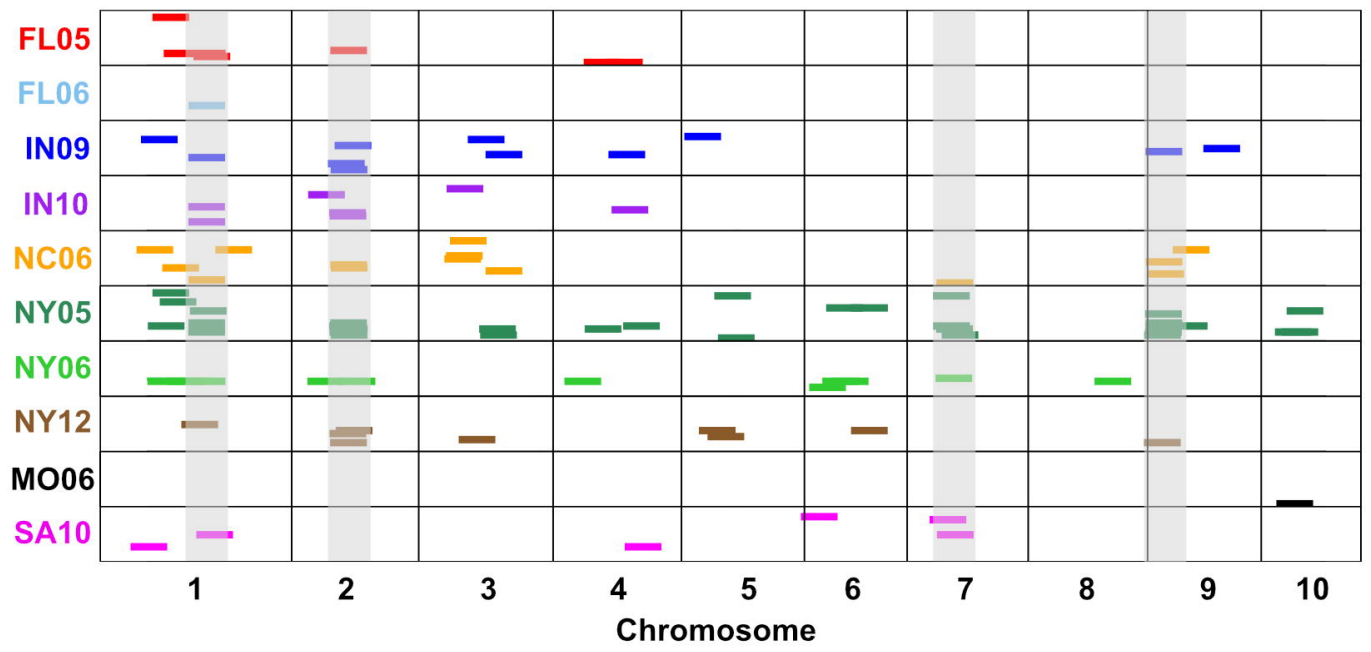




\section{A}

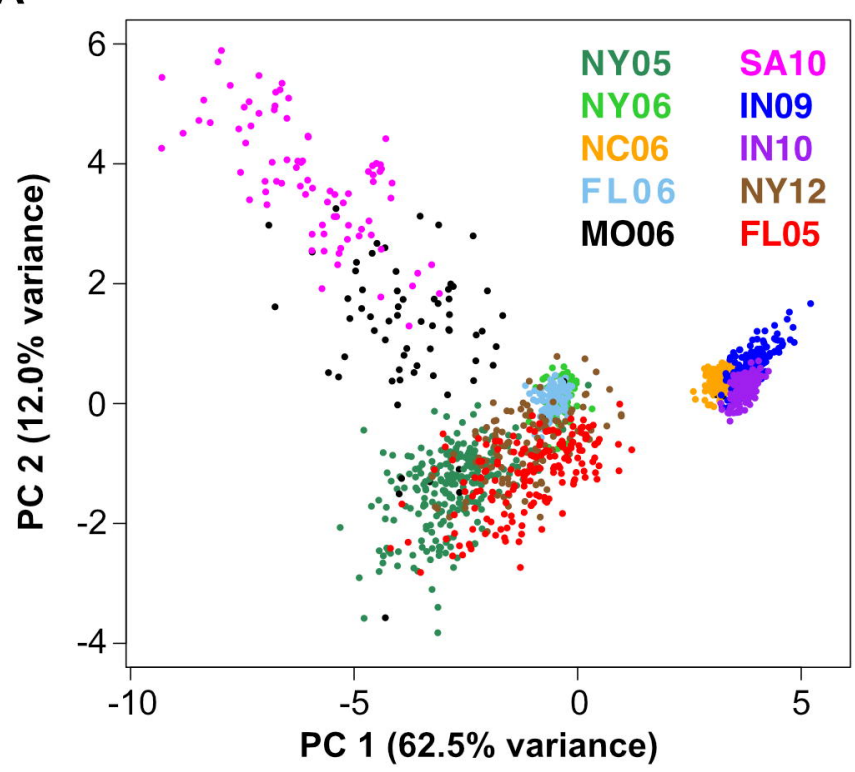

B

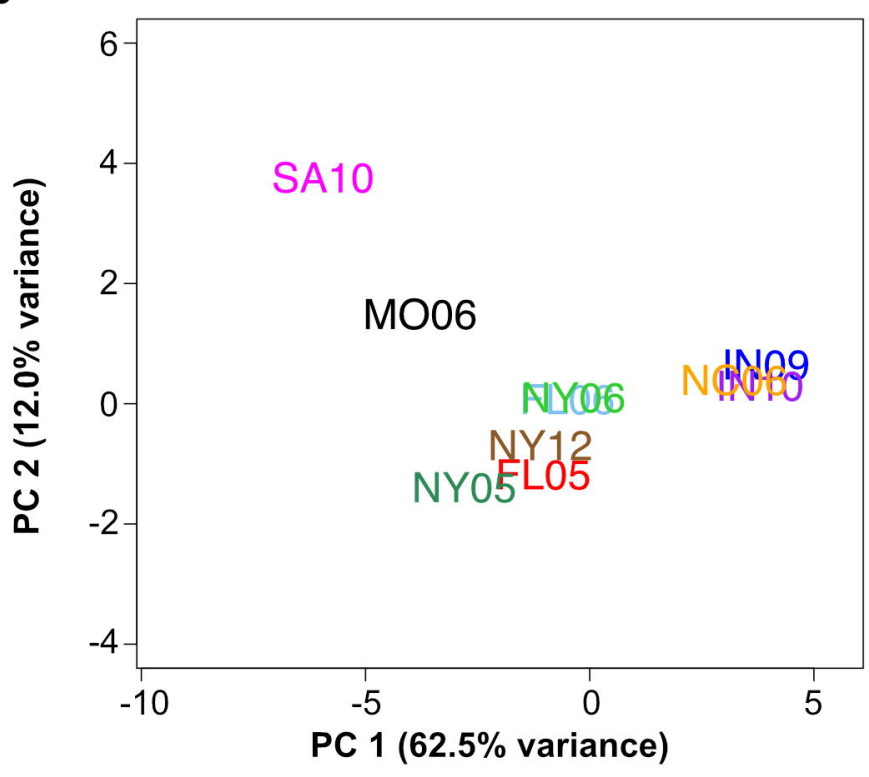




\section{A Weather Data and aPC 1-7 Correlations}

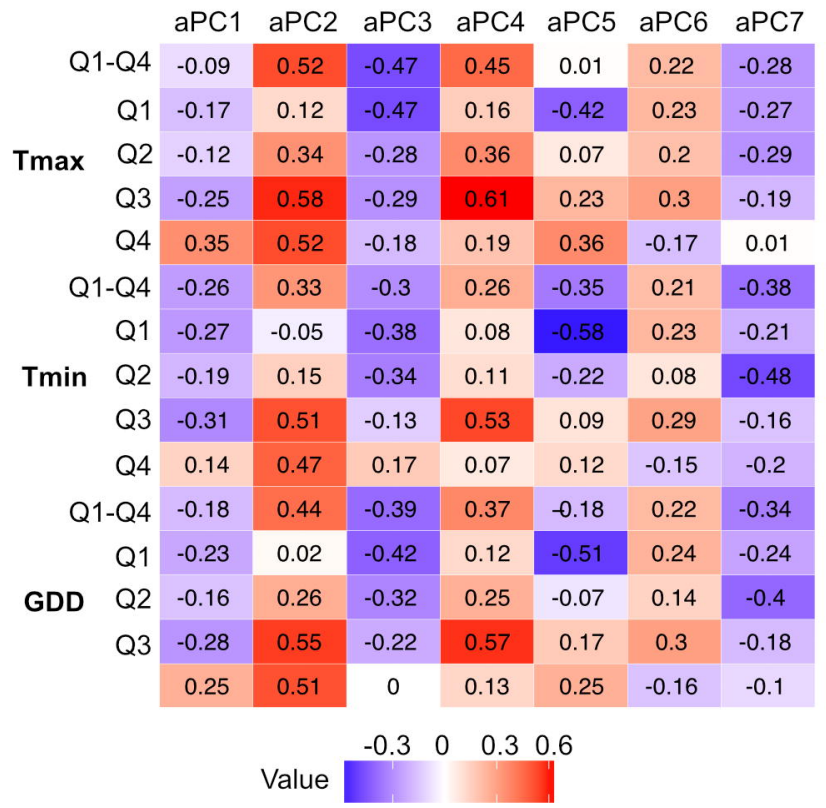

D

Soil Data and aPC 1-7 Correlations

\begin{tabular}{|c|c|c|c|c|c|c|c|}
\hline & $\mathrm{aPC} 1$ & aPC2 & aPC3 & $\mathrm{aPC} 4$ & aPC5 & aPC6 & aPC7 \\
\hline $\mathrm{pH}$ & -0.27 & & 0.43 & -0.42 & -0.31 & -0.17 & 0.12 \\
\hline EC & -0.19 & -0.33 & -0.14 & -0.3 & -0.59 & -0.09 & -0.28 \\
\hline AWC & -0.28 & 0.47 & -0.11 & 0.65 & 0.21 & 0.36 & 0.11 \\
\hline AWS & -0.08 & 0.32 & 0.12 & 0.45 & 0.41 & 0.2 & 0.28 \\
\hline $\mathrm{CaCO} 3$ & -0.23 & -0.48 & -0.01 & -0.35 & -0.58 & -0.11 & -0.21 \\
\hline
\end{tabular}

Value $\quad \begin{array}{lll}-0.4 & 0 & 0.4 \\ & & \end{array}$

\section{B}

aPC1 vs 4th Quarter Tmax

30 $r_{p}=.35$

Avg 28

Tmax (deg

C)

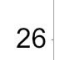

24.

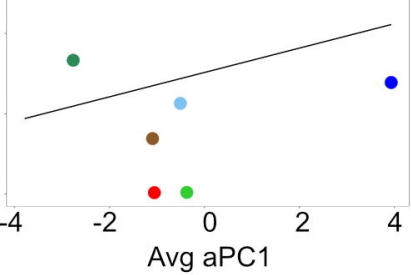

C

aPC2 vs 3rd Quarter Tmax $r_{p}=.58$

Avg

Tmax

(deg

C)

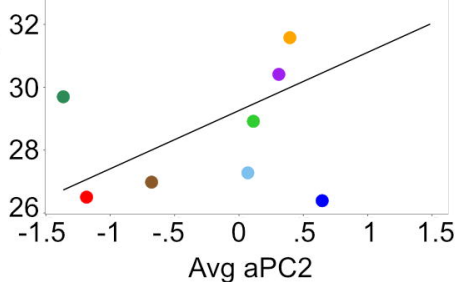

E

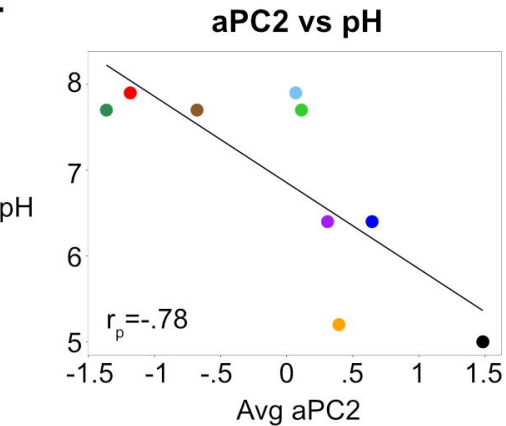




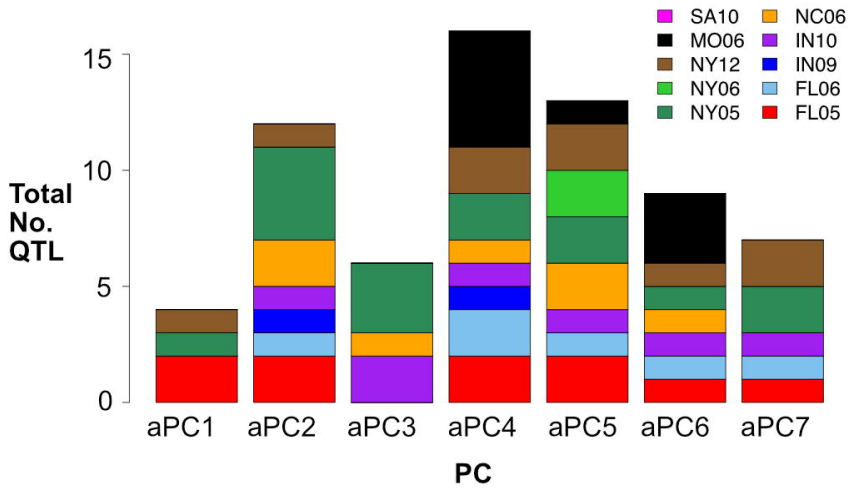

Full Genome Plot: PCA Across Environments QTL

\begin{tabular}{|c|c|c|c|c|c|c|c|c|c|c|}
\hline $\mathrm{aPC} 7$ & - & - & & & - & & & & & \\
\hline aPC6 & - & - & & - & & & & - & & \\
\hline aPC5 & - & - & & & & - & & - & & \\
\hline $\mathrm{aPC} 4$ & - & - & & - & & & $=$ & - & & \\
\hline aPC3 & - & - & & & & & - & & & \\
\hline aPC2 & - & - & & -- & & & & & - & \\
\hline aPC1 & & & & - & - & & - & & & \\
\hline & 1 & 2 & 3 & 4 & 5 & 6 & 7 & 8 & 9 & 10 \\
\hline
\end{tabular}

